\title{
Sharp Hardy inequalities in an exterior of a ball
}

\author{
Nikolai Kutev* Tsviatko Rangelov *
}

\begin{abstract}
New Hardy type inequalities in sectorial area and as a limit in an exterior of a ball are proved. Sharpness of the inequalities is shown as well.
\end{abstract}

Keywords: Hardy inequalities, sharp estimates.

2010 Mathematics Subject Classification: 26D10, 35P15

\section{Introduction}

The classical Hardy inequality, proved in Hardy [7, 8] states

$$
\int_{0}^{\infty}\left|u^{\prime}(x)\right|^{p} x^{\alpha} d x \geq\left(\frac{p-1-\alpha}{p}\right)^{p} \int_{0}^{\infty} x^{-p+\alpha}|u(x)|^{p} d x
$$

where $1<p<\infty, \alpha<p-1$ and $u(x)$ is absolutely continuous on $[0, \infty), u(0)=0$.

There are several generalizations of (1) in multidimensional case mostly in bounded domains, see Davies [4], Ghoussoub and Moradifam [6], Balinsky et al. [3], Kutev and Rangelov [9] and the literature therein. For unbounded domains, in the exterior of a boll, only few generalizations of (1) are reported in Wang and Zhu [10], Adimurthi et al. [1], Avkhadiev and Laptev [2].

For example, in Theorem 1.1 in Wang and Zhu [10] for all function $u \in D_{a}^{1,2}\left(R^{n}\right)$, where $D_{a}^{1,2}\left(R^{n}\right)$ is the weighted Sobolev space - the comletion of $C_{0}^{\infty}\left(R^{n}\right)$ with the norm $\int_{R^{n}}|x|^{-2 a}|u|^{2} d x$, the following inequality for $a<\frac{n-2}{2}$ is proved

$$
\int_{B_{1}^{c}}|x|^{-2 a}|\nabla u|^{2} d x \geq\left(\frac{n-2-2 a}{2}\right)^{2} \int_{B_{1}^{c}} \frac{|u|^{2}}{|x|^{2}(a+1)} d x+\frac{n-2-2 a}{2} \int_{\partial B_{1}^{c}} u^{2},
$$

where $B_{1}^{c}=\{|x|>1\}$.

Let us mention that in Wang and Zhu [10] Hardy inequalities with weights in unbounded domains $\Omega \subset R^{n}, 0 \notin \partial \Omega$ are also considered, see Theorem 1.3 and Remark 1.5 .

In Adimurthi et al. [1], Corollary, for $n \geq 3$ and $u \in C_{0}^{\infty}\left(B_{1}^{c}\right)$ the limiting case of the Caffarelli-Kohn-Nirenberg inequality

$$
\int_{B_{1}^{c}}|\nabla u|^{2} d x \geq\left(\frac{n-2}{2}\right)^{2} \int_{B_{1}^{c}} \frac{u^{2}}{|x|^{2}} d x+C_{n}(a)\left[\int_{B_{1}^{c}} X_{1}^{\frac{2(n-1)}{n-2}}\left(a, \frac{1}{|x|}\right) u^{\frac{2 n}{n-2}} d x\right]^{\frac{n-2}{n}},
$$

\footnotetext{
*Institute of Mathematics and Informatics, Bulgarian Academe of Sciences, 1113, Sofia, Bulgaria Corresponding author: T. Rangelov, rangelov@math.bas.bg
} 
is proved, where $\left.X_{1}(a, s)=a-\ln s\right)^{-1}, a>0,0<s \leq 1$ and the constant $C_{n}(a)$ is given explicitly.

Finally, in Corollary 1 and Remark 1 in Avkhadiev and Laptev [2] the following Hardy inequality is proved for $n \geq 3$ and $u \in W_{0}^{1,2}\left(B_{r}^{c}\right)$

$$
\int_{B_{r}^{c}}|\nabla u|^{2} d x \geq\left(\frac{n-2}{2}\right)^{2} \int_{B_{r}^{c}} \frac{u^{2}}{|x|^{2}} d x+\frac{1}{4} \int_{B_{r}^{c}}\left(\frac{1}{|x-r|^{2}}-\frac{1}{|x|^{2}}\right) u^{2} d x .
$$

At the end of the paper we compare the inequalities (2), (3), (4) with our results.

The aim of the present work is to derive new Hardy inequalities in the exterior of a ball. There are listed also functions for which these inequalities are sharp, i.e., inequalities with an optimal constant of the leading term become equations.

\section{Inequalities in sectorial area}

We start with Hardy inequalities in sectorial area $B_{R} \backslash B_{r}$ where $B_{R}, B_{r}$ are balls centered at zero, $0<r<R$. Let $1<p, p^{\prime}=\frac{p}{p-1}, 2 \leq n$ and denote $m=\frac{p-n}{p-1}=\frac{p-n}{p} p^{\prime}$.

For functions $u$ such that $\int_{B_{R} \backslash B_{r}}\left|\frac{\langle x, \nabla u\rangle}{|x|}\right|^{p} d x<\infty$ let us define two sets

$$
\begin{aligned}
& M_{1}(r, R)=\left\{\begin{array}{l}
\left|\frac{R^{m}-\hat{R}^{m}}{m}\right|^{1-p} \int_{\partial B_{\hat{R}}}|u|^{p} d \sigma \rightarrow 0, \quad \hat{R} \rightarrow R-0, \quad m \neq 0, \\
\left|\ln \frac{R}{\hat{R}}\right|^{1-n} \int_{\partial B_{\hat{R}}}|u|^{n} d \sigma \rightarrow 0, \quad \hat{R} \rightarrow R-0, \quad m=0
\end{array}\right. \\
& M_{2}(r, R)=\left\{\begin{array}{l}
\left|\frac{\hat{r}^{m}-r^{m}}{m}\right|^{1-p} \int_{\partial B_{\hat{r}}}|u|^{p} d \sigma \rightarrow 0, \quad \hat{r} \rightarrow r+0, \quad m \neq 0, \\
\left|\ln \frac{\hat{r}}{r}\right|^{1-n} \int_{\partial B_{\hat{r}}}|u|^{n} d \sigma \rightarrow 0, \quad \hat{r} \rightarrow r+0, \quad m=0
\end{array}\right.
\end{aligned}
$$

where $\langle$,$\rangle is a scalar product in R^{n}$.

Let functions $\psi_{j}$ be solutions of the problems:

$$
\begin{aligned}
& -\Delta_{p} \psi_{1}=0, \quad \text { in } B_{R} \backslash \bar{B}_{r},\left.\quad \psi_{1}\right|_{\partial B_{R}}=0,\left.\quad \psi_{1}\right|_{\partial B_{r}}=1, \\
& -\Delta_{p} \psi_{2}=0, \quad \text { in } B_{R} \backslash \bar{B}_{r},\left.\quad \psi_{2}\right|_{\partial B_{R}}=1,\left.\quad \psi_{2}\right|_{\partial B_{r}}=0 .
\end{aligned}
$$

Their explicit form is:

$$
\psi_{1}(x)=\left\{\begin{array}{l}
\frac{R^{m}-|x|^{m}}{R^{m}-r^{m}}, \quad m \neq 0, \\
\frac{\ln \frac{R}{|x|}}{\ln \frac{R}{r}}, m=0
\end{array} \quad, \quad \psi_{2}(x)=\left\{\begin{array}{l}
\frac{|x|^{m}-r^{m}}{R^{m}-r^{m}}, \quad m \neq 0, \\
\frac{\ln \frac{|x|}{r}}{\ln \frac{R}{r}}, m=0
\end{array} .\right.\right.
$$


We can define vector functions $f_{i}$ as $f_{i}=\left|\frac{\nabla \psi_{i}}{\psi_{i}}\right|^{p-2} \frac{\nabla \psi_{i}}{\psi_{i}}$. in $B_{R} \backslash \bar{B}_{r}$ and

$$
\begin{aligned}
L^{i}(u) & =\int_{B_{R} \backslash B_{r}}\left|\frac{\left\langle\nabla \psi_{i}, \nabla u\right\rangle}{\left|\nabla \psi_{i}\right|}\right|^{p} d x=\int_{B_{R} \backslash B_{r}}\left|\frac{\langle x, \nabla u\rangle}{|x|}\right|^{p} d x, \\
K^{i}(u) & =\int_{B_{R} \backslash B_{r}}\left|\frac{\nabla \psi_{i}}{\psi_{i}}\right|^{p}|u|^{p} d x=\int_{B_{R} \backslash B_{r}}\left|f_{i}\right|^{p^{\prime}}|u|^{p} d x, \\
K_{0}^{i}(u) & =\int_{\partial\left(B_{R} \backslash B_{r}\right)}\left|\frac{\nabla \psi_{i}}{\psi_{i}}\right|^{p-2}\left\langle\frac{\nabla \psi_{i}}{\psi_{i}}, \nu\right\rangle|u|^{p} d s=\int_{\partial\left(B_{R} \backslash B_{r}\right)}\left\langle f_{i}, \nu\right\rangle|u|^{p} d x .
\end{aligned}
$$

Here $\nu$ is the outward normal to $B_{R} \backslash B_{r}$.

The following Theorem takes place.

Theorem 2.1. For every $u \in M_{i}(r, R)$ we have

$$
L^{i}(u) \geq\left(\frac{1}{p}\right)^{p} \frac{\left[K_{0}^{i}(u)+(p-1) K_{1}^{i}(u)\right]^{p}}{\left(K_{1}^{i}(u)\right)^{p-1}}=K^{i}(u) .
$$

where $\nu$ is the outward normal to $B_{R} \backslash B_{r}$.

Proof. We follow the proof of Proposition 1 in Fabricant et al. [5]. Since

$$
\int_{B_{\hat{R}} \backslash B_{\hat{r}}}\left\langle f_{i}, \nabla|u|^{p}\right\rangle d x=p \int_{B_{\hat{R}} \backslash B_{\hat{r}}}|u|^{p-2} u\left\langle f_{i}, \nabla u\right\rangle d x
$$

where $r<\hat{r}<\hat{R}<R$. Then applying Hölder inequality on the $r h s$ of (7) with $\frac{\langle x, \nabla u\rangle}{|x|}$ and $\left|f_{i}\right||u|^{p-2} u$ as factors of the integrand we get

$$
\begin{aligned}
\int_{B_{\hat{R}} \backslash B_{\hat{r}}}\left\langle f_{i}, \nabla|u|^{p}\right\rangle d x & \leq p\left(\int_{B_{\hat{R}} \backslash B_{\hat{r}}}\left|\frac{\langle x, \nabla u\rangle}{|x|}\right|^{p} d x\right)^{1 / p} \\
& \times\left(\int_{B_{\hat{R}} \backslash B_{\hat{r}}}\left|f_{i}\right|^{p^{\prime}}|u|^{p} d x\right)^{1 / p^{\prime}} .
\end{aligned}
$$

Rising to $p$ power both sides of (8) it follows that

$$
\int_{B_{\hat{R}} \backslash B_{\hat{r}}}\left|\frac{\langle x, \nabla u\rangle}{|x|}\right|^{p} d x \geq \frac{\left|\frac{1}{p} \int_{B_{\hat{R}} \backslash B_{\hat{r}}}\left\langle f_{i}, \nabla|u|^{p}\right\rangle d x\right|^{p}}{\left(\int_{B_{\hat{R}} \backslash B_{\hat{r}}}\left|f_{i}\right|^{p^{\prime}}|u|^{p} d x\right)^{p-1}} .
$$

Integrating by parts the numerator of the right hand side of (9) we get

$$
\begin{aligned}
& \frac{1}{p} \int_{B_{\hat{R}} \backslash B_{\hat{r}}}\left\langle f_{i}, \nabla|u|^{p}\right\rangle d x=\frac{1}{p} \int_{\partial B_{\hat{R}} \cup \partial B_{\hat{r}}}\left\langle f_{i}, \nu\right\rangle|u|^{p} d S-\frac{1}{p} \int_{B_{\hat{R}} \backslash B_{\hat{r}}} \operatorname{div} f_{i}|u|^{p} d x \\
& =\frac{1}{p} \int_{\partial B_{\hat{R}} \cup \partial B_{\hat{r}}}\left\langle f_{i}, \nu\right\rangle|u|^{p} d S+\left(\frac{p-1}{p}\right) \int_{B_{\hat{R}} \backslash B_{\hat{r}}}|f|^{p^{\prime}}|u|^{p} d x \\
& \rightarrow \frac{1}{p}\left((p-1) K^{i}+K_{0}^{i}\right), \text { when } \hat{R} \rightarrow R-0, \quad \hat{r} \rightarrow r+0 .
\end{aligned}
$$


Note that $\int_{\partial B_{R} \cup \partial B_{r}}\left\langle f_{i}, \nu\right\rangle|u|^{p} d S \geq 0$ for $u \in M_{i}(r, R)$, since $\left.\nu\right|_{\partial B_{R}}=\left.\frac{x}{|x|}\right|_{\partial B_{R}},\left.\nu\right|_{\partial B_{r}}=$ $-\left.\frac{x}{|x|}\right|_{\partial B_{r}}$. From (9) and (10) we obtain (6) since

$$
-\operatorname{div} f_{i}=(p-1)\left|f_{i}\right|^{p^{\prime}}
$$

\section{Inequalities in an exterior of a ball $B_{r}^{c}=R^{n} \backslash \bar{B}_{r}$}

Let us introduce functions $u$ such that

$$
\int_{B_{r}^{c}}\left|\frac{<x, \nabla u>}{|x|}\right|^{p} d x<\infty, \quad \int_{B_{r}^{c}} \frac{|u|^{p}}{|x|^{(n-1) p^{\prime}}} d x<\infty
$$

and define two sets

$$
\begin{aligned}
& M_{1}(r, \infty)=\left\{\begin{array}{l}
R^{1-n} \int_{\partial B_{R}}|u|^{p} d \sigma \rightarrow 0, \quad R \rightarrow \infty, \quad m \geq 0, \\
R^{1-p} \int_{\partial B_{R}}|u|^{p} d \sigma \rightarrow 0, \quad R \rightarrow \infty, \quad m<0 .
\end{array}\right. \\
& M_{2}(r, \infty)=\left\{\begin{array}{l}
\left|\frac{\hat{r}^{m}-r^{m}}{m}\right|^{1-p} \int_{\partial B_{\hat{r}}}|u|^{p} d \sigma \rightarrow 0, \quad \hat{r} \rightarrow r+0, \quad m \neq 0, \\
\left(\ln \frac{\hat{r}}{r}\right)^{1-p} \int_{\partial B_{\hat{r}}}|u|^{p} d \sigma \rightarrow 0, \quad \hat{r} \rightarrow r+0, \quad m=0 .
\end{array}\right.
\end{aligned}
$$

In a similar way we can prove Theorem 2.1, replacing $B_{R} \backslash \bar{B}_{r}$ with $B_{r}^{c}=R^{n} \backslash \bar{B}_{r}$ and $\partial\left(B_{R} \backslash \bar{B}_{r}\right)$ with $\partial B_{r}^{c}=\partial B_{r}$ and $L^{i}(u), K_{0}^{I}(u), K_{1}^{i}(u)$ define in (5) for $R \rightarrow \infty$. The inequalities below for functions of $M_{i}(r, \infty), i=1,2$ can be obtained with a limit $R \rightarrow \infty$.

Proposition 3.1. For every $u \in M_{1}(r, \infty)$ the following inequalities hold:

$$
\begin{aligned}
& \left(\int_{B_{r}^{c}} \frac{|u|^{p}}{|x|^{(n-1) p^{\prime}}} d x\right)^{\frac{1}{p^{\prime}}}\left(\int_{B_{r}^{c}}\left|\frac{<x, \nabla u>}{|x|}\right|^{p} d x\right)^{\frac{1}{p}} \\
& \geq \frac{1}{p} r^{1-n} \int_{\partial B_{r}}|u|^{p} d S, \text { for } m>0 .
\end{aligned}
$$

With function $u_{\alpha}(x)=e^{-\alpha|x|^{m}}, \quad \alpha>0$ inequality (11) becomes equality.

(ii) For $m<0$ we get:

$$
\begin{aligned}
& \left(\int_{B_{r}^{c}}\left|\frac{<x, \nabla u>}{|x|}\right|^{p} d x\right)^{\frac{1}{p}} \geq \frac{|m|}{p^{\prime}}\left(\int_{B_{r}^{c}} \frac{|u|^{p}}{|x|^{p}} d x\right)^{\frac{1}{p}} \\
& +\frac{1}{p} r^{1-p} \int_{\partial B_{r}}|u|^{p} d S\left(\int_{B_{r}^{c}} \frac{|u|^{p}}{|x|^{p}} d x\right)^{-\frac{1}{p^{\prime}}}, \text { for } m<0 .
\end{aligned}
$$

With function $u_{k}(x)=|x|^{k m}, \quad k>p^{\prime}$ inequality (12) becomes an equality. 
(iii)

$$
\begin{aligned}
& \left(\int_{B_{r}^{c}} \frac{|u|^{n}}{|x|^{n}} d x\right)^{\frac{n-1}{n}}\left(\int_{B_{r}^{c}}\left|\frac{<x, \nabla u>}{|x|}\right|^{n} d x\right)^{\frac{1}{n}} \\
& \geq \frac{1}{n} r^{1-n} \int_{\partial B_{r}}|u|^{n} d S, \text { for } m=0 .
\end{aligned}
$$

With function $u_{q}(x)=|x|^{q}, \quad q<0$ inequality (13) becomes equality.

Proof. For $m \neq 0$ the inequality (6) has the form

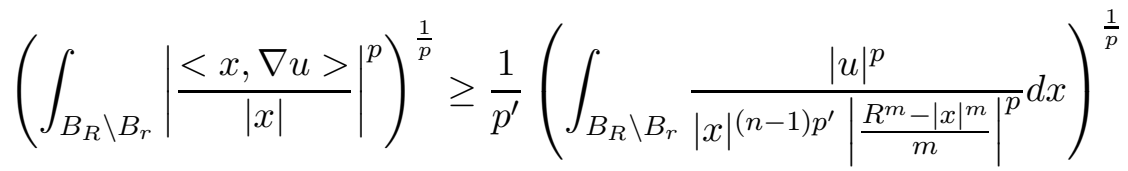

$$
\begin{aligned}
& +\frac{1}{p} r^{1-n}\left|\frac{R^{m}-r^{m}}{m}\right|^{1-p} \int_{\partial B_{r}}|u|^{p} d S\left(\int_{B_{R} \backslash B_{r}} \frac{|u|^{p}}{|x|^{(n-1) p^{\prime}}\left|\frac{R^{m}-|x|^{m}}{m}\right|^{p}} d x\right)^{-\frac{1}{p^{\prime}}} .
\end{aligned}
$$

Analogously, for $m=0$, i. e. $p=n$ the inequality (6) becomes

$$
\begin{aligned}
& \left(\int_{B_{R} \backslash B_{r}}\left|\frac{<x, \nabla u>}{|x|}\right|^{n} d x\right)^{\frac{1}{n}} \geq \frac{n-1}{n}\left(\int_{B_{R} \backslash B_{r}} \frac{|u|^{n}}{|x|^{n}\left|\ln \frac{R}{|x|}\right|^{n}} d x\right)^{\frac{1}{n}} \\
+ & \frac{1}{n}\left(r \ln \frac{R}{r}\right)^{1-n} \int_{\partial B_{r}}|u|^{n} d S\left(\int_{B_{R} \backslash B_{r}} \frac{|u|^{n}}{\left.|x|^{n} \mid \ln \frac{R}{|x|^{n}} d x\right)^{\frac{1-n}{n}} .}\right.
\end{aligned}
$$

(i) For $m>0$, after the limit $R \rightarrow \infty$ in (14) we obtain

$$
\begin{aligned}
& \frac{1}{p^{\prime}}\left(\int_{B_{R} \backslash B_{r}} \frac{|u|^{p}}{|x|^{(n-1) p^{\prime}}\left|\frac{R^{m}-|x|^{m}}{m}\right|^{p}} d x\right)^{\frac{1}{p}} \rightarrow_{R \rightarrow \infty} 0 \text { and } \\
& \frac{1}{p} r^{1-n}\left|\frac{R^{m}-r^{m}}{m}\right|^{1-p} \int_{\partial B_{r}}|u|^{p} d S\left(\int_{B_{R} \backslash B_{r}} \frac{|u|^{p}}{|x|^{(n-1) p^{\prime}}\left|\frac{R^{m}-|x|^{m}}{m}\right|^{p}} d x\right)^{-\frac{1}{p^{\prime}}} \\
& \rightarrow R \rightarrow \infty \\
& \frac{1}{p} r^{1-n} \int_{\partial B_{r}}|u|^{p} d S\left(\int_{B_{r}^{c}} \frac{|u|^{p}}{|x|^{(n-1) p^{\prime}}} d x\right)^{-\frac{1}{p^{\prime}}} \cdot
\end{aligned}
$$

and hence (11) holds.

Let us check that with the function $u_{\alpha}(x)=e^{-\alpha|x|^{m}}, \alpha>0$ inequality (11) becomes an 
equality. Simply computation gives us

$$
\begin{aligned}
I_{1 \alpha} & =\left(\int_{B_{r}^{c}} \frac{e^{-\alpha p|x|^{m}}}{|x|^{(n-1) p^{\prime}}} d x\right)^{\frac{1}{p^{\prime}}}=\left(\int_{S_{1}} \int_{r}^{\infty} e^{-\alpha p \rho^{m}} \rho^{-(n-1) p^{\prime}} \rho^{n-1} d \rho d S\right)^{\frac{1}{p^{\prime}}} \\
& =\left|S_{1}\right|^{\frac{1}{p^{\prime}}}\left(\frac{1}{m} \int_{r}^{\infty} e^{-\alpha p \rho^{m}} d \rho^{m}\right)^{\frac{1}{p^{\prime}}}=\left|S_{1}\right|^{\frac{1}{p^{\prime}}} \frac{1}{(\alpha p m)^{\frac{1}{p^{\prime}}}} e^{-\alpha(p-1) r^{m}}, \\
I_{2 \alpha} & =\left(\int_{B_{r}^{c}}\left|\frac{<x, \nabla u>}{|x|}\right|^{p} d x\right)^{\frac{1}{p}}=\left(\int_{S_{1}} \int_{r}^{\infty} e^{-\alpha p \rho^{m}}(\alpha m)^{p} \rho^{-(n-1) p^{\prime}} \rho^{n-1} d \rho d S\right)^{\frac{1}{p}} \\
& =\left|S_{1}\right|^{\frac{1}{p}}\left(\frac{(\alpha m)^{p}}{m \alpha p} \int_{r}^{\infty} e^{-\alpha p \rho^{m}} d \rho^{m}\right)^{\frac{1}{p}}=\left|S_{1}\right|^{\frac{1}{p}} \frac{\alpha m}{(\alpha p m)^{\frac{1}{p}}} e^{-\alpha r^{m}}, \\
I_{3 \alpha} & =\frac{1}{p} r^{1-n} \int_{\partial B_{r}}\left|u_{\alpha}\right|^{p} d S=\frac{1}{p} r^{1-n} \int_{S_{1}} e^{-\alpha p r^{m}} r^{n-1} d S=\left|S_{1}\right| \frac{1}{p} e^{-\alpha p r^{m}},
\end{aligned}
$$

and we get the equality $I_{1 \alpha} I_{2 \alpha}=I_{3 \alpha}$.

(ii) For $m<0$, after the limit $R \rightarrow \infty$ in (14) we obtain (12). Since $k>\frac{1}{p^{\prime}}$ it follows that $u_{k}(x) \in M_{1}(r, \infty)$. Moreover, inequality (12) becomes equality. Indeed,

$$
\begin{aligned}
I_{1 k} & =\left(\int_{B_{r}^{c}}\left|\frac{<x, \nabla u_{k}>}{|x|}\right|^{p} d x\right)^{\frac{1}{p}}=\left(\left.\left.\int_{B_{r}^{c}}|k m| x\right|^{k m-1}\right|^{p} d x\right)^{\frac{1}{p}} \\
& =|k m|\left(\left|S_{1}\right| \int_{r}^{\infty} \rho^{(k m-1) p} \rho^{n-1} d \rho\right)^{\frac{1}{p}}=|k m|\left(\left|S_{1}\right| \frac{r^{(k m-1) p+n}}{|(k m-1) p+n|}\right)^{\frac{1}{p}}, \\
I_{2 k} & =\frac{|m|}{p^{\prime}}\left(\int_{B_{r}^{c}} \frac{\left|u_{k}\right|^{p}}{|x|^{p}} d x\right)^{\frac{1}{p}}=\frac{|m|}{p^{\prime}}\left(\left|S_{1}\right| \int_{r}^{\infty} \rho^{(k m-1) p+n-1} d \rho\right)^{\frac{1}{p}} \\
& =\frac{|m|}{p^{\prime}}\left(\left|S_{1}\right| \frac{r^{(k m-1) p+n}}{|(k m-1) p+n|}\right)^{\frac{1}{p}}, \\
I_{3 k} & =\frac{1}{p} r^{1-p} \int_{\partial B_{r}}\left|u_{k}\right|^{p} d S\left(\int_{B_{r}^{c}} \frac{\left|u_{k}\right|^{p}}{|x|^{p}} d x\right)^{-\frac{1}{p^{\prime}}} \\
& =\frac{1}{p} r^{1-p}\left|S_{1}\right| r^{k m p+n-1}\left(\left|S_{1}\right| \frac{r^{(k m-1) p+n}}{(k m-1) p+n}\right)^{-\frac{1}{p^{\prime}}} \\
& =\frac{1}{p}\left(\left|S_{1}\right| r^{|(k m-1) p+n|}\right)^{\frac{1}{p}}(|(k m-1) p+n|)^{\frac{1}{p^{\prime}}} .
\end{aligned}
$$

Since

$$
\frac{|k m|}{|(k m-1) p+n|}=\frac{|m|}{p^{\prime}} \frac{1}{|(k m-1) p+n|}+\frac{1}{p}
$$


we get the equality $I_{1 k}=I_{2 k}+I_{3 k}$.

(iii) For $m=0$, after the limit $R \rightarrow \infty$ in (15), since

$$
\begin{aligned}
& \frac{n-1}{n}\left(\int_{B_{R} \backslash B_{r}} \frac{|u|^{n}}{|x|^{n}\left|\ln \frac{R}{|x|}\right|^{n}} d x\right)^{\frac{1}{n}} \rightarrow_{R \rightarrow \infty} 0 \text { and } \\
& \frac{1}{n}\left(r \ln \frac{R}{r}\right)^{1-n} \int_{\partial B_{r}}|u|^{n} d S\left(\int_{B_{R} \backslash B_{r}} \frac{|u|^{n}}{|x|^{n}\left|\ln \frac{R}{|x|}\right|^{n}} d x\right)^{\frac{1-n}{n}} \\
& =\frac{1}{n} r^{1-n} \ln ^{1-n} R\left(1-\frac{\ln r}{\ln R}\right)^{1-n} \int_{\partial B_{r}}|u|^{n} d S \frac{1}{\ln ^{1-n} R}\left(\int_{B_{R} \backslash B_{r}} \frac{|u|^{n}}{|x|^{n}\left|1-\frac{\ln |x|}{\ln R}\right|^{n}} d x\right)^{\frac{1-n}{n}} \\
& \rightarrow R \rightarrow \infty \frac{1}{n} r^{1-n} \int_{\partial B_{r}}|u|^{n} d S\left(\int_{B_{r}^{c}} \frac{|u|^{n}}{|x|^{n}} d x\right)^{\frac{1-n}{n}}
\end{aligned}
$$

we obtain (13). Let us check that with function $u_{q}(x)=|x|^{q}, q<0$ inequality (13) becomes equality. Indeed,

$$
\begin{aligned}
& I_{1 q}=\left(\int_{B_{r}^{c}} \frac{\left|u_{q}\right|^{n}}{|x|^{n}} d x\right)^{\frac{n-1}{n}}=\left(\left|S_{1}\right| \int_{r}^{\infty} \rho^{(q-1) n} \rho^{n-1} d \rho\right)^{\frac{n-1}{n}}=\left|S_{1}\right|^{\frac{n-1}{n}}(n|q|)^{-\frac{n-1}{n}} r^{(n-1) q}, \\
& I_{2 q}=\left(\int_{B_{r}^{c}}\left|\frac{<x, \nabla u_{q}>}{|x|}\right|^{n} d x\right)^{\frac{1}{n}}=\left(\left|S_{1}\right| \int_{r}^{\infty}|q|^{n} \rho^{(q-1) n} \rho^{n-1} d \rho\right)^{\frac{1}{n}}=\left|S_{1}\right|^{\frac{1}{n}}|q|^{\frac{n-1}{n}} n^{-\frac{1}{n}} r^{q}, \\
& I_{3 q}=\frac{1}{n} r^{1-n} \int_{\partial B_{r}}\left|u_{q}\right|^{n} d S=\frac{1}{n} r^{1-n}\left|S_{1}\right| r^{n q} r^{n-1}=\left|S_{1}\right| \frac{1}{n} r^{q n},
\end{aligned}
$$

and we get the equality $I_{1 q} I_{2 q}=I_{3 q}$.

Proposition 3.2. For every $u \in M_{2}(r, \infty)$ the following inequalities hold:

(i)

$$
\begin{aligned}
& \quad\left(\int_{B_{r}^{c}} \mid \frac{<x, \nabla u>\left.\right|^{p}}{|x|} d x\right)^{\frac{1}{p}} \geq \frac{m}{p^{\prime}}\left(\int_{B_{r}^{c}} \frac{|u|^{p}}{\left.|x|^{\left.\left.(n-1) p^{\prime}\left|r^{m}-\right| x\right|^{m}\right|^{p}} d x\right)^{\frac{1}{p}}}\right. \\
& +\frac{1}{p} \limsup _{R \rightarrow \infty}\left[R^{1-p} \int_{\partial B_{R}}|u|^{p} d S\right]\left(\int_{B_{r}^{c}} \frac{|u|^{p}}{\left.|x|^{(n-1) p^{\prime}}|| x\right|^{m}-\left.r^{m}\right|^{p}} d x\right)^{-\frac{1}{p^{\prime}}}, \\
& \quad \text { for } m>0 .
\end{aligned}
$$


With function $u_{\varepsilon}(x)=|x|^{-\frac{m(1-\varepsilon)}{p^{\prime}}}\left(|x|^{m}-r^{m}\right)^{\frac{(1+\varepsilon)}{p^{\prime}}}, 0<\varepsilon<1$ inequality (16) is $\varepsilon$-sharp, i.e.

$$
\left(\frac{m}{p^{\prime}}\right)^{p} \leq \frac{L^{1}\left(u_{\varepsilon}\right)}{\left[\left(K^{1}\left(u_{\varepsilon}\right)\right)^{\frac{1}{p}}+K_{0}^{1}\left(u_{\varepsilon}\right)\left(K^{1}\left(u_{\varepsilon}\right)\right)^{-\frac{1}{p^{\prime}}}\right]^{p}} \leq \frac{L^{1}\left(u_{\varepsilon}\right)}{K^{1}\left(u_{\varepsilon}\right)} \leq\left(\frac{m}{p^{\prime}}\right)^{p}(1+\varepsilon)^{p} .
$$

(ii)

$$
\begin{aligned}
& \left(\int_{B_{r}^{c}}\left|\frac{<x, \nabla u>}{|x|}\right|^{p} d x\right)^{\frac{1}{p}} \geq \frac{|m|}{p^{\prime}}\left(\int_{B_{r}^{c}} \frac{|u|^{p}}{\left.|x|^{\left.\left.(n-1) p^{\prime}\left|r^{m}-\right| x\right|^{m}\right|^{p}} d x\right)^{\frac{1}{p}}}\right. \\
& +\frac{r^{n-p}}{p} \limsup _{R \rightarrow \infty}\left[R^{1-n} \int_{\partial B_{R}}|u|^{p} d S\right]\left(\int_{B_{r}^{c}} \frac{|u|^{p}}{\left.|x|^{(n-1) p^{\prime}}|| x\right|^{m}-\left.r^{m}\right|^{p}} d x\right)^{-\frac{1}{p^{\prime}}}, \\
& \quad \text { for } m<0 .
\end{aligned}
$$

With function $u_{s}(x)=\left(r^{m}-|x|^{m}\right)^{s}, \quad s>\frac{1}{p^{\prime}}$ inequality (18) becomes equality.

(iii)

$$
\begin{aligned}
& \left(\int_{B_{r}^{c}}\left|\frac{<x, \nabla u>}{|x|}\right|^{n} d x\right)^{\frac{1}{n}} \geq \frac{n-1}{n}\left(\int_{B_{r}^{c}} \frac{|u|^{n}}{|x|^{n}\left|\ln \frac{|x|}{r}\right|^{n}} d x\right)^{\frac{1}{n}} \\
& +\frac{1}{n} \limsup _{R \rightarrow \infty}\left[\left(R \ln \frac{R}{r}\right)^{1-n} \int_{\partial B_{R}}|u|^{n} d S\right]\left(\int_{B_{r}^{c}} \frac{|u|^{n}}{|x|^{n}\left|\ln \frac{|x|^{n}}{r}\right|^{n}} d x\right)^{\frac{1-n}{n}}, \\
& \quad \text { for } m=0 .
\end{aligned}
$$

For function

$$
u_{\eta}(x)=\left\{\begin{array}{ll}
\left(\ln \frac{|x|}{r}\right)^{\frac{n-1}{n}\left(1+\frac{\eta}{2}\right)} M^{\frac{n-1}{n}\left(1-\frac{\eta}{2}\right)}, \quad r<|x|<M \\
\left(\ln \frac{|x|}{r}\right)^{\frac{n-1}{n}\left(1+\frac{\eta}{2}\right)}|x|^{\frac{n-1}{n}\left(1-\frac{\eta}{2}\right)}, \quad M<|x|
\end{array},\right.
$$

where $0<\eta<1$, inequality (19) is $\eta$-sharp, i.e.

$$
\left(\frac{n-1}{n}\right)^{n} \leq \frac{L^{1}\left(u_{\eta}\right)}{K^{1}\left(u_{\eta}\right)} \leq\left(\frac{n-1}{n}\right)^{n}(1+\eta)^{n}
$$


Proof. For $m \neq 0$ inequality (6) has the form

$$
\begin{gathered}
\left(\int_{B_{R} \backslash B_{r}}\left|\frac{<x, \nabla u>}{|x|}\right|^{p}\right)^{\frac{1}{p}} \geq \frac{|m|}{p^{\prime}}\left(\int_{B_{R} \backslash B_{r}} \frac{|u|^{p}}{\left.|x|^{\left.\left.(n-1) p^{\prime}\left|r^{m}-\right| x\right|^{m}\right|^{p}} d x\right)^{\frac{1}{p}}}\right. \\
+\frac{1}{p} R^{1-n}\left|R^{m}-r^{m}\right|^{1-p} \int_{\partial B_{R}}|u|^{p} d S\left(\int_{B_{R} \backslash B_{r}} \frac{|u|^{p}}{\left.|x|^{\left.\left.(n-1) p^{\prime}\left|r^{m}-\right| x\right|^{m}\right|^{p}} d x\right)^{-\frac{1}{p^{\prime}}} .}\right.
\end{gathered}
$$

while for $m=0$ (6) becomes

$$
\begin{aligned}
& \left(\int_{B_{R} \backslash B_{r}}\left|\frac{<x, \nabla u>}{|x|}\right|^{n} d x\right)^{\frac{1}{n}} \geq \frac{n-1}{n}\left(\int_{B_{R} \backslash B_{r}} \frac{|u|^{n}}{|x|^{n}\left|\ln \frac{|x|}{r}\right|^{n}} d x\right)^{\frac{1}{n}} \\
+ & \frac{1}{n}\left(R \ln \frac{R}{r}\right)^{1-n} \int_{\partial B_{r}}|u|^{n} d S\left(\int_{B_{R} \backslash B_{r}} \frac{|u|^{n}}{|x|^{n} \mid \ln \frac{|x|^{n}}{r}} d x\right)^{\frac{-1}{n^{\prime}}} .
\end{aligned}
$$

(i) If $m>0$ after the limit $R \rightarrow \infty$ in (21) we obtain

$$
\begin{aligned}
& \frac{1}{p} \limsup _{R \rightarrow \infty}\left[R^{1-n}\left|R^{m}-r^{m}\right|^{1-p} \int_{\partial B_{R}}|u|^{p} d S\right] \\
& =\frac{1}{p} \limsup _{R \rightarrow \infty}\left[R^{1-n} R^{n-p}\left|1-\frac{r^{m}}{R^{m}}\right|^{1-p} \int_{\partial B_{R}}|u|^{p} d S\right] \\
& =\frac{1}{p} \limsup _{R \rightarrow \infty} R^{1-p} \int_{\partial B_{R}}|u|^{p} d S
\end{aligned}
$$

which proves (16).

For the function $u_{\varepsilon}(x)=|x|^{-\frac{m(1-\varepsilon)}{p^{\prime}}}\left(|x|^{m}-r^{m}\right)^{\frac{(1+\varepsilon)}{p^{\prime}}}, 0<\varepsilon<1, u_{\varepsilon}(x) \in M_{2}(r, \infty)$ 
simple computation give us

$$
\begin{aligned}
& I_{1 \varepsilon}=L^{1}\left(u_{\varepsilon}\right)=\int_{B_{r}^{c}}\left|\frac{\left\langle x, \nabla u_{\varepsilon}>\right.}{|x|}\right|^{p} d x \\
& =\left(\frac{m}{p^{\prime}}\right)^{p} \int_{B_{r}^{c}}|x|^{-m(1-\varepsilon)(p-1)-p}\left(|x|^{m}-r^{m}\right)^{(1+\varepsilon(p-1)-p} \\
& \times\left[(1+\varepsilon)|x|^{m}-(1-\varepsilon)\left(|x|^{m}-r^{m}\right)\right]^{p} d x \\
& =\left(\frac{m}{p^{\prime}}\right)^{p}\left|S_{1}\right| \int_{r}^{\infty}\left(\rho^{m}-r^{m}\right)^{(1+\varepsilon)(p-1)-p} \\
& \times\left[(1+\varepsilon) \rho^{m}-(1-\varepsilon)\left(\rho^{m}-r^{m}\right)\right]^{p} \rho^{-m(1-\varepsilon)(p-1)-p+n-1} d \rho \\
& \leq\left(\frac{m}{p^{\prime}}\right)^{p}\left|S_{1}\right|(1+\varepsilon)^{p} \int_{r}^{\infty}\left(\rho^{m}-r^{m}\right)^{(1+\varepsilon)(p-1)-p} \rho^{-m(1-\varepsilon)(p-1)-p+n-1+m p} d \rho \\
& =\left(\frac{m}{p^{\prime}}\right)^{p}\left|S_{1}\right|(1-\varepsilon)^{p} \int_{r}^{\infty}\left(\rho^{m}-r^{m}\right)^{(1+\varepsilon)(p-1)-p} \rho^{-m(1+\varepsilon)(p-1)-\frac{n-1}{p-1}} d \rho,
\end{aligned}
$$

because $n-1-p+m p=-\frac{n-1}{p-1}$.

$$
\begin{aligned}
I_{2 \varepsilon} & =K^{1}\left(u_{\varepsilon}\right)=\int_{B_{r}^{c}}|x|^{-m(1-\varepsilon)(p-1)-(n-1) p^{\prime}}\left(|x|^{m}-r^{m}\right)^{(1+\varepsilon)(p-1)-p} d x \\
& =\left|S_{1}\right| \int_{r}^{\infty}\left(\rho^{m}-r^{m}\right)^{(1+\varepsilon)(p-1)-p} \rho^{-m(1-\varepsilon)(p-1)-\frac{n-1}{p-1}} d \rho .
\end{aligned}
$$

Thus (17) follows immediately from expressions of $I_{1 \varepsilon}$ and $I_{2 \varepsilon}$. (ii) When $m<0$, after the limit $R \rightarrow \infty$ in (21) we get

$$
\begin{aligned}
& \frac{1}{p} \limsup _{R \rightarrow \infty}\left[R^{1-n}\left|R^{m}-r^{m}\right|^{1-p} \int_{\partial B_{R}}|u|^{p} d S\right] \\
& =\frac{1}{p} \limsup _{R \rightarrow \infty}\left[r^{n-p} R^{1-n}\left|\frac{R^{m}}{r^{m}}-1\right|^{1-p} \int_{\partial B_{R}}|u|^{p} d S\right] \\
& =\frac{r^{n-p}}{p} \limsup _{R \rightarrow \infty} R^{1-n} \int_{\partial B_{R}}|u|^{p} d S
\end{aligned}
$$

which proves (18). 
For the function $u_{s}(x)=\left(r^{m}-|x|^{m}\right)^{s}, s>\frac{1}{p^{\prime}}$ we have the identities

$$
\begin{aligned}
I_{1 s} & =\left(\int_{B_{r}^{c}}\left|\frac{<x, \nabla u_{s}>}{|x|}\right|^{p} d x\right)^{\frac{1}{p}}=\left(s^{p}|m|^{p}\left|S_{1}\right| \int_{r}^{\infty} \rho^{p(m-1)+n-1}\left(r^{m}-\rho^{m}\right)^{(s-1) p} d \rho\right)^{\frac{1}{p}} \\
& =s|m|\left|S_{1}\right|^{\frac{1}{p}}\left(-\frac{1}{m} \int_{r}^{\infty}\left(r^{m}-\rho^{m}\right)^{(s-1) p} d\left(r^{m}-\rho^{m}\right)\right)^{\frac{1}{p}}, \\
& =s|m|^{\frac{1}{p^{\prime}}}\left|S_{1}\right|^{\frac{1}{p}} r^{\frac{m}{p}[(s-1) p+1]}[(s-1) p+1]^{-\frac{1}{p}}
\end{aligned}
$$

because $p(m-1)+n-1=m-1$.

$$
\begin{aligned}
& I_{2 s}=\int_{B_{r}^{c}} \frac{\left|u_{s}\right|^{p}}{\left.\left.|x|^{(n-1) p^{\prime}}\left|r^{m}-\right| x\right|^{m}\right|^{p}} d x=\left|S_{1}\right| \int_{r}^{\infty} \rho^{(n-1)\left(1-p^{\prime}\right)}\left|r^{m}-\rho^{m}\right|^{(s-1) p} d \rho \\
& =\frac{\left|S_{1}\right|}{|m|} \frac{r^{m[(s-1) p+1]}}{(s-1) p+1}, \\
& I_{3 s}=\frac{r^{n-p}}{p} \limsup _{R \rightarrow \infty}\left[R^{1-n} \int_{\partial B_{R}}\left|r^{m}-R^{m}\right|^{s p} d S\right] \\
& =\frac{r^{n-p}}{p} \limsup _{R \rightarrow \infty}\left|R^{m}-r^{m}\right|^{s p}\left|S_{1}\right|=\frac{\left|S_{1}\right|}{p} r^{n-p+m p s} .
\end{aligned}
$$

Simple computation gives us the equality

$$
\begin{aligned}
& \frac{|m|}{p^{\prime}}\left(I_{2 s}\right)^{\frac{1}{p}}+I_{3 s}\left(I_{2 s}\right)^{-\frac{1}{p^{\prime}}} \\
& =\frac{\left|S_{1}\right||m|^{\frac{1}{p^{\prime}}} r^{\frac{m}{p}[(s-1) p+1]}}{p^{\prime}[(s-1) p+1]^{\frac{1}{p}}}+\frac{\left|S_{1}\right| r^{n-p+m p s}}{p}\left(\frac{\left|S_{1}\right|}{|m|}\right)^{-\frac{1}{p^{\prime}}}\left[\frac{r^{m[(s+1) p+1]}}{(s-1) p+1}\right]^{-\frac{1}{p^{\prime}}} \\
& =\frac{1}{p}|m|^{\frac{1}{p^{\prime}}}\left|S_{1}\right|^{\frac{1}{p}} r^{\frac{m}{p}[(s-1) p+1]}\left(\frac{p-1}{[(s-1) p+1]^{p}}+[(s-1) p+1]^{\frac{1}{p^{\prime}}}\right) \\
& =s|m|^{\frac{1}{p^{\prime}}}\left|S_{1}\right|^{\frac{1}{p}} r^{\frac{m}{p}[(s-1) p+1]}[(s-1) p+1]^{-\frac{1}{p}}=I_{1 s}
\end{aligned}
$$

because

$$
n-p+m p s-\frac{m}{p^{\prime}}[(s-1) p+1]=\frac{m}{p}[(s-1) p+1]
$$

(iii) After the limit $R \rightarrow \infty$ in (22) we get (19).

The function $u_{\eta}(x)$ belongs to $M_{2}(r, \infty)$ for $m=0$. Moreover, for $r<|x|<M$ we have the equalities

$$
\begin{aligned}
& I_{1 \eta}=L^{1}\left(u_{\eta}\right)=M^{(n-1)(1-\eta)}\left(1+\frac{\eta}{2}\right)^{n}\left(\frac{n-1}{n}\right)^{n}\left|S_{1}\right| \int_{r}^{M}\left(\ln \frac{\rho}{r}\right)^{(n-1)\left(1+\frac{\eta}{2}\right)-n} \rho^{-1} d \rho, \\
& I_{2 \eta}=K^{1}\left(u_{\eta}\right)=M^{(n-1)\left(1+\frac{\eta}{2}\right)}\left|S_{1}\right| \int_{r}^{M}\left(\ln \frac{\rho}{r}\right)^{(n-1)\left(1+\frac{\eta}{2}\right)-n} \rho^{-1} d \rho,
\end{aligned}
$$


and hance

$$
\frac{L^{1}\left(u_{\eta}\right)}{K^{1}\left(u_{\eta}\right)}=\left(\frac{n-1}{n}\right)^{n}\left(1+\frac{\eta}{2}\right)^{n} \leq\left(\frac{n-1}{n}\right)^{n}(1+\eta)^{n} .
$$

Tedious calculations give us for $|x| \geq M$ the identities

$$
\begin{aligned}
& I_{1 \eta}=L^{1}\left(u_{\eta}\right)=\left(\frac{n-1}{n}\right)^{n}\left|S_{1}\right| \int_{M}^{\infty} \rho^{(n-1)\left(1-\frac{\eta}{2}\right)-1}\left(\ln \frac{\rho}{r}\right)^{(n-1)\left(1+\frac{\eta}{2}\right)-n} \\
& \times\left[\left(1-\frac{\eta}{2}\right) \frac{1}{\rho} \ln \frac{\rho}{r}+\left(1+\frac{\eta}{2}\right)\right]^{n} d \rho \\
& I_{2 \eta}=K^{1}\left(u_{\eta}\right)=\left|S_{1}\right| \int_{r}^{M} \rho^{(n-1)\left(1-\frac{\eta}{2}-1\right.}\left(\ln \frac{\rho}{r}\right)^{(n-1)\left(1+\frac{\eta}{2}\right)-n}
\end{aligned}
$$

If $M$ is sufficiently large, i.e.,

$$
\frac{1}{M} \ln \frac{M}{r}<\frac{\eta}{2-\eta} \quad \text { and } \quad M>e r
$$

then

$$
\left(1-\frac{\eta}{2}\right) \frac{1}{\rho} \ln \frac{\rho}{r}+1+\frac{\eta}{2} \leq\left(1-\frac{\eta}{2}\right) \frac{\eta}{2-\eta}+1+\frac{\eta}{2}=1+\eta,
$$

because the function $h(\rho)=\frac{1}{\rho} \ln \frac{\rho}{r}$ is monotone decreasing for $\rho>$ er. Thus (20) follows from expressions of $I_{1 \eta}$ and $I_{2 \eta}$ above for $|x| \geq r$.

\section{Sharp inequalities in an exterior of a ball $B_{r}^{c}$ for $u \in$ $W_{0}^{1, p}\left(B_{r}^{c}\right)$}

For $m<0$, i.e. $p<n$, we can combine inequalities (14) and (12) for functions $u \in M(r, \infty)$, where

$$
M(r, \infty)=\left\{\begin{array}{l}
u \in W_{0}^{1, p}\left(B_{r}^{c}\right), \\
\left(\frac{\hat{r}^{m}-r^{m}}{m}\right)^{1-p} \int_{\partial B_{\hat{r}}}|u|^{p} d x \rightarrow 0, \quad \hat{r} \rightarrow r+0, \\
R^{1-p} \int_{\partial B_{R}}|u|^{p} d x \rightarrow 0, \quad \text { for } R \rightarrow \infty .
\end{array}\right.
$$

For $r<\gamma<\infty, \gamma=2^{\frac{1}{|m|}} r$, we define

$$
\begin{aligned}
& L_{1}(u)=\int_{B_{\gamma} \backslash B_{r}}\left|\frac{\langle x, \nabla u\rangle}{|x|}\right|^{p} d x, \quad L_{2}(u)=\int_{B_{\gamma}^{c}}\left|\frac{\langle x, \nabla u\rangle}{|x|}\right|^{p} d x, \\
& K_{11}(u)=|m|^{p} \int_{B_{\gamma} \backslash B_{r}} \frac{|u|^{p}}{|x|^{(n-1) p^{\prime}}\left(|x|^{m}-r^{m}\right)^{p}} d x, \quad K_{12}(u)=|m|^{p} \int_{B_{\gamma}^{c}} \frac{|u|^{p}}{|x|^{p}} d x \\
& K_{01}(u)=|m|^{p-1} \gamma^{1-p} \int_{\partial B_{\gamma}}|u|^{p} d \sigma=K_{02}(u)=|m|^{p-1} \gamma^{1-p} \int_{\partial B_{\gamma}}|u|^{p} d \sigma=K_{0} .
\end{aligned}
$$


Proposition 4.1. If $\gamma=2^{1 /|m|} r$ then for every $u \in M(r, \infty)$ the inequality

$$
L(u)=\sum_{1}^{2} L_{j}(u)=\int_{B_{r}^{c}}\left|\frac{\langle x, \nabla u\rangle}{|x|}\right|^{p} d x \geq\left(\frac{1}{p}\right)^{p} \sum_{1}^{2} \frac{\left[K_{0 j}(u)+(p-1) K_{1 j}(u)\right]^{p}}{\left(K_{1 j}(u)\right)^{p-1}}=K(u),
$$

holds in $B_{r}^{c}$.

The inequality (23) becomes an equality for functions $u_{\beta}(x) \in M(r, \infty), \beta>1 / p^{\prime}$ where

$$
u_{\beta}(x)=\left\{\begin{array}{l}
\left(r^{m}-|x|^{m}\right)^{\beta} \quad x \in B_{\gamma} \backslash B_{r}, \\
|x|^{m \beta}, \quad x \in B_{\gamma}^{c}
\end{array}\right.
$$

Proof. As in Theorem 2.1 and Proposition 3.1 ii) we get

$$
\begin{aligned}
& L_{1}(u) \geq K_{1}(u) \quad \text { in } \quad B_{\gamma} \backslash B_{r}, \\
& L_{2}(u) \geq K_{2}(u) \quad \text { in } \quad B_{\gamma}^{c}
\end{aligned}
$$

where

$$
K_{j}(u)=\frac{\left[K_{0 j}(u)+(p-1) K_{1 j}(u)\right]^{p}}{\left(K_{1 j}(u)\right)^{p-1}} .
$$

With the choice of $\gamma=2^{1 /|m|} r$, so $\gamma^{p}=\gamma^{(n-1) p^{\prime}}\left(r^{m}-\gamma^{m}\right)^{p}$, we have continuous kernel for $K_{j 1}$ on $\partial B_{\gamma}$.

Adding inequalities in (24) we get for $u \in M(r, \infty) L(u)=\sum_{1}^{2} L_{j}(u) \geq \sum_{1}^{2} K_{j}(u)$. Hance from the Joung inequality we obtain (23)

Using (12) in $B_{\gamma}^{c}$ and (15) in $B_{\gamma \backslash r}$ it follows that the inequality (23) becomes equality for functions $u_{\beta}(x), \beta>\frac{1}{p^{\prime}}$.

Proposition 4.1 gives sharp Hardy inequality in an exterior of a ball $B_{r}^{c}$ for functions $u \in W_{0}^{1, p}\left(B_{r}^{c}\right), p<n$.

We will illustrate Proposition 3.2 ii) and Proposition 4.1 in the following examples.

Example 4.1. For $p=2, n \geq 3, r=1, a=0$ and $m=2-n<0$ from Proposition 3.1 ii) it follows that the Hardy inequality

$$
\int_{B_{1}^{c}}|\nabla u|^{2} d x \geq\left(\frac{n-2}{2}\right)^{2} \int_{B_{1}^{c}} \frac{|u|^{2}}{|x|^{2}} d x+\frac{n-2}{2} \int_{\partial B_{1}^{c}} u^{2}+\frac{1}{4} \int_{\partial B_{1}^{c}} u^{2}\left(\int_{B_{1}^{c}} \frac{|u|^{2}}{|x|^{2}}\right)^{-1},
$$

holds. Note that (25) has an additional term in the right hand side in comparison with (2). Moreover, for the functions $u_{k}(x)=|x|^{k(2-n)}, k>2$ inequality (25) becomes an equality, i.e., inequality (25) is sharp. 
Example 4.2. For $p=2, n \geq 3, m=2-n<0, \gamma=2^{\frac{1}{n-2}} r$, and every function $u \in$ $W_{0}^{1,2}\left(B_{r}^{c}\right)$ the Hardy inequality $(24)$ becomes

$$
\begin{aligned}
& \left.\int_{B_{r}^{c}} \frac{\langle x, \nabla u\rangle}{|x|}\right|^{p} d x \geq\left(\frac{n-2}{2}\right)^{2} \int_{B_{r}^{c}} \frac{u^{2}}{|x|^{2}} d x \\
& +2\left(\frac{n-2}{2}\right)^{2} \int_{B_{\gamma} \backslash B_{r}} \frac{\left|\frac{x}{r}\right|^{n-2}\left(1-\left|\frac{x}{\gamma}\right|^{n-2}\right)}{\left(\left|\frac{x}{r}\right|^{n-2}-1\right)^{2}} u^{2} d x+\frac{2^{-\frac{1}{n-2}}}{r}(n-2) \int_{\partial B_{\gamma}} u^{2} d S \\
& +\frac{2^{-\frac{2}{n-2}}}{r^{2}}\left(\frac{4}{n-2}\right)^{2}\left(\int_{\partial B_{\gamma}} u^{2} d S\right)^{2} \\
& \times\left[\left(\int_{B_{\gamma} \backslash B_{r}} \frac{u^{2}}{|x|^{2}\left(\left|\frac{x}{r}\right|^{n-2}-1\right)^{2}} d x\right)^{-2}+\left(\int_{B_{\gamma}^{c}} \frac{u^{2}}{|x|^{2}} d x\right)^{-2}\right]
\end{aligned}
$$

Moreover, for function $u_{\beta}(x)$ defined in Proposition 4.1 for $m=2-n$, inequality (26) becomes equality.

Let us mention that Hardy inequality (26) has the same leading term in the right hand side as in inequality (3), but inequality (26) is sharp one.

Finally, it is difficult to compare inequality (4) with (26), but (26) is sharp one, i.e., for the functions $u_{\beta}(x)$ defined in Proposition 4.1, inequality (26) becomes an equality.

Acknowledgement This paper is partially supported by the National Scientific Program "Information and Communication Technologies for a Single Digital Market in Science, Education and Security (ICTinSES)", contract No DO1205/23.11.2018, financed by the Ministry of Education and Science in Bulgaria and also by the Grant No BG05M2OP001-1.001-0003, financed by the Science and Education for Smart Growth Operational Program (2014-2020) in Bulgaria and cofinanced by the European Union through the European Structural and Investment Funds.

\section{References}

[1] Adimurthi, S. Filippas, and A. Tertikas. On the best constant of Hardy-Sobolev inequalities. Nonlinear Anal. T.M.A., 70:2826-2833, 2009.

[2] F. Avkhadiev and A. Laptev. Hardy inequalities for nonconvex domains. In International Mathematical Series, vol 11, Around Research of Vladimir Maz'ya, I, pages 1-12, Springer, New York, 2010.

[3] A. A. Balinsky, W. D. Evans, and R. T. Lewis. The analysis and geometry of Hardy's Inequality. Springer Int. Publisher, Cham, 2015.

[4] E. Davies. A review of Hardy inequalities. Oper. Theory Adv. Appl., 110:57-67, 1998.

[5] A. Fabricant, N. Kutev, and T. Rangelov. Sharp hardy inequalities in a ball. Pliska Stud. Math. Bulgar, 23:67-80, 2014.

[6] N. Ghoussoub and A. Moradifam. Functional inequalities: New perspectives and new applications. AMS, Math. Surveys and Monographs, v. 187, Rhode Island, 2013. 
[7] G. Hardy. Note on a theorem of Hilbert. Math. Zeit., 6:314-317, 1920.

[8] G. Hardy. Notes on some points in the integral calculus, LX. an inequality between integrals. Messenger of Math., 54:150-156, 1925.

[9] N. Kutev and T. Rangelov. Hardy inequalities with double singular weights. 2020. arXiv.2001.07368v2.

[10] Z-Q. Wang and M. Zhu. Hardy inequalities with boundary terms. Electron. J. Diff. Eq., 43: $1-8,2003$. 